\title{
Mechanical Characteristics of Basic Actions in Simulated Space Operation Conditions
}

\author{
Fan $\mathrm{Li}^{1, \mathrm{a}}{ }^{\mathrm{a}}$ Chunhui Wang ${ }^{1, \mathrm{~b}}$, Haoting $\mathrm{Liu}^{1}$, Zheng Wang ${ }^{1}$, Ming $\mathrm{Iv}^{2}$ \\ ${ }^{1}$ Science and Technology on Human Factors Engineering Laboratory, China Astronaut Research \\ and Training Center, Beijing, China \\ ${ }^{2}$ General Hospital of Armed Police Forces, Beijing, China \\ aEmail: lifan_miao@live.cn, ${ }^{\text {b}}$ Email: chunhui_89@yahoo.com.cn
}

Keywords: mechanical characteristics; astronauts operation experiment; extravehicular suit; suspended condition; Hybridized K-means algorithm

\begin{abstract}
The objective of this paper is to elucidate how the ergonomic experiment is designed to simulate the space operation conditions and evaluate the influence of these conditions on mechanical characteristics of basic actions. The ergonomic methodologies and data analysis technologies are translated into a potential for better understanding of astronauts' potential incapacitation during space flight. Firstly, the ergonomic simulation experiment is implemented to get the operation force data of basic actions. Secondly, the force data are classified and identified from different operation conditions by the Hybridized K-means method. Finally, the analysis results are utilized to evaluate the condition effect on mechanical characteristics. It shows that the data of suspended conditions affect upper limb strength more significantly than the result of the constrained condition. The Hybridized K-means algorithm is a good attempt of the evaluation methods for the assessment of the astronaut operation capabilities.
\end{abstract}

\section{Introduction}

In Chinese space station program, astronauts or engineers, who engage in space science research in internal or external station, will implement a series of in-orbit activities including space survival, instrument operation, physical exercise, etc. In addition to the changes in the physical and psychological factors, some external factors influence the completion quality of space tasks, such as the microgravity environment, operation posture, mode of operation, etc. For example, the change of astronaut operation posture will lead to the changes in the relative positions of the various body limbs and lower limb coordination. [5] So we must analyze the mechanical characteristics of the astronaut space operation in order to assist the space task design and the optimization of the operation procedures.

The number of past experiments related to understanding how the astronauts' awareness of operation position changes during space flight was very small for the limited time and space. The experiments on the ground could not eliminate gravity effort and simulate appropriate operation conditions with poor equipments. But many processes of space tasks have shown that our knowledge of the mechanical characteristics of the astronaut space operation may be altered dramatically. Astronauts have difficulty in positioning their lower limbs precisely by themselves during "drop tests" in space. [7] Others have shown the difficulty of maintaining a body position perpendicular to the vehicle floor (an "upright posture") by their feet only and estimating the angle of their knees and elbows when tested repeatedly in space. [6]

From the perspective of mechanics, whatever tasks and actives the astronauts perform, these operations are reflected statics or dynamic responses with different postures and motions. And they can be divided into a few basic actions, such as: push, pull, twist, etc. Therefore, in this study, we initially carry out an ergonomic experiment of basic actions and approximately simulate astronaut space operation conditions on the ground. By comparative analysis of the mechanical characteristics of operation force data obtained under different experimental conditions, we verify the analyzing 
method for an attempt to build a preliminary analysis model. Based on the analyzing results, we also give some ergonomic suggestions in order to help astronauts finish this kind of tasks with efficiency.

In the following sections, the design of experiments will be illustrated, and then the experimental operation force data will be analyzed by the Hybridized K-means algorithm. Finally, some discussions and suggestions will be presented.

\section{Materials and Methods}

The purpose of the experiments is to simulate space operation conditions step by step and analyze the mechanical characteristics of different kinds of force data in different operation conditions with consistency.

Subjects. 15 young male volunteers (age: $22.1 \pm 2.1$ years, height: $176.3 \pm 2.3 \mathrm{~cm}$, and weight: $68.9 \pm 3.4 \mathrm{~kg}$ ) participate in this experiment. They are in good health, no muscle fatigue, and no strenuous exercise in 24 hours before the experiment. And they agree and are familiar with a variety of experimental methods and requirements.

Experimental Method. We simulate astronaut space operation conditions approximately to study the mechanical changes of basic actions. With our existing experimental facilities, we have designed four kinds of experimental conditions.

a) Constrained condition without extravehicular suit (Condition I): The subject holds two mechanical data acquisition handles (Fig. 1a) in the fixed slide-rails on the wall and gets his feet into a foot restraint with two foot plate mechanical data acquisitions. (Fig. 1b) When the subject operates, the handled and foot restraint will be locked.

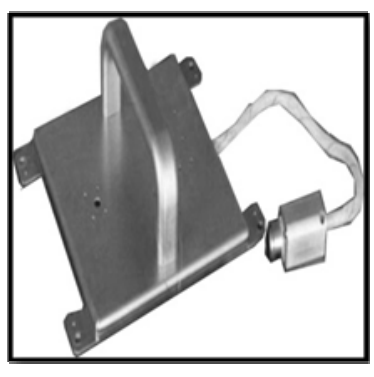

(a)

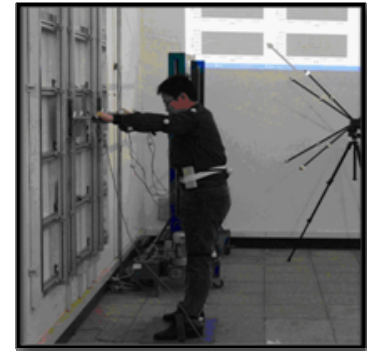

(b)

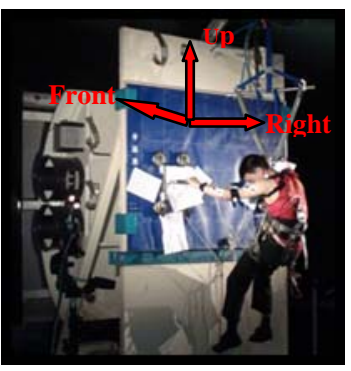

(c)

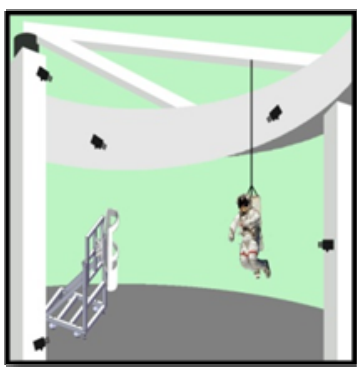

(d)

Figure 1. Sketch of experiment

b) Suspended condition without extravehicular suit (Condition II): The subject is suspended by suspension system that is mainly composed by two air flotation rails. (Fig. 1d) The mechanical data acquisition handles with sucking discs can be adjusted the position in a smooth plexiglass plate. And the plexiglass plate is in fixed shelf that can be adjusted its height. (Fig. 1c)

c) Constrained condition in extravehicular suit (Condition III): The subject, who is in extravehicular suit and gets his feet into a foot restraint with two foot plate mechanical data acquisitions (introduced in Condition II), operates the mechanical data acquisition handles (introduced in Condition I ) in fixed extravehicular suit shelf that can be adjusted its height

d) Suspended conditon in extravehicular suit (Condition IV): The subject, who is suspended in and extravehicular suit, is measured his operation force data and uses the same devices introduced in Condition II . (Fig. 1d)

In each operation condition, operation positions are based on operation height and operation width. Operation height is divided into 110CM, 140CM, and 170CM from the subject's soles of his feet, and operation width is divided into $35 \mathrm{CM}, 55 \mathrm{CM}$, and $75 \mathrm{CM}$, which is the distance between the subject's two hands. So the total operation positions are nine kinds. At each position, the directions of basic actions are same and listed in Table 1. The frame of reference is subject himself. (Fig. 1c)

Table 1 Directions of Basic Actions

\begin{tabular}{|c|c|c|c|c|c|c|c|}
\hline Basic & \multicolumn{2}{|c|}{ Directions } & Repeat & Basic & \multicolumn{2}{|c|}{ Directions } & Repeat \\
\cline { 2 - 6 } Action & Left Hand & Right Hand & Time & Action & Left Hand & Right Hand & Time \\
\hline 1 & Left & Left & 3 & 7 & Front & Back & 3 \\
\hline
\end{tabular}




\begin{tabular}{|c|c|c|c|c|c|c|c|}
\hline 2 & Right & Right & 3 & 8 & Back & Front & 3 \\
\hline 3 & Right & Left & 3 & 9 & Up & Up & 3 \\
\hline 4 & Left & Right & 3 & 10 & Down & Down & 3 \\
\hline 5 & Front & Front & 3 & 11 & Up & Down & 3 \\
\hline 6 & Back & Back & 3 & 12 & Down & Up & 3 \\
\hline
\end{tabular}

Each kind of basic action is repeated three times with maximal strength for about 5 seconds at intervals of 15 seconds. The subject will have one minute rest after each kind of basic actions. So the subject should complete each kind of condition experiment at twice and have at least three days of rest after each implement in order to alleviate his muscle fatigue. Every subject implements eight times to finish the entire experiment totally.

Hybridized K-means Clustering Algorithm. K-means clustering algorithm (K-means) is one of the major data analysis methods, which is widely used for many practical applications in emerging areas like Bioinformatics [1]. Traditional K-means is commonly used partitioning based clustering technique that tries to find a user specified number of clusters $(k)$, which are represented by their centroids, by minimizing the square error function [2]. And it for cluster analysis developed for low dimensional data, often do not work well for high dimensional data and the results may not be accurate most of the time due to noise and outliers associated with original data [3]. Our operation force signal data of experiments have many dimensions, included different hands, directions, and axes and noises. Hence, to improve the efficiency, we have applied a kind of hybridized K-means clustering algorithm [4], which is mixed the Principal Component Analysis (PCA) method on original data set, so that the correlated variables exist in the original dataset will be transformed to possibly uncorrelated variables, which are reduced in size.

\section{Results and Discussions}

Results. For precise operation force calculation, the statistical force of basic actions was the average value of the same kind of operation action, repeated by three times. By set $K=4$, the statistical operation force of Condition I , II, III, and IV, were processed by the hybridized Kmeans in Table 2 partly. All given cluster of operation force data was partitioned into 4 clusters. The cluster recognition rates were accurate as much as possible and the inter distances between operation force data clusters in different conditions were maintained to be as large as possible.

Table 2 The Statistical Value of Operation Force in Four Conditions by K-means (K=4)

\begin{tabular}{|c|c|c|c|c|c|c|c|c|c|}
\hline \multirow{9}{*}{ 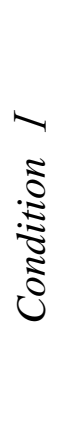 } & Source & Class & Source & Class & \multirow{9}{*}{ 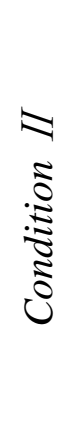 } & Source & Class & Source & Class \\
\hline & Subject01 & 2 & Subject09 & 2 & & Subject01 & 4 & Subject09 & 3 \\
\hline & Subject02 & 2 & Subject10 & 2 & & Subject02 & 4 & Subject10 & 3 \\
\hline & Subject03 & 2 & Subject11 & 4 & & Subject03 & 3 & Subject11 & 4 \\
\hline & Subject04 & 2 & Subject12 & 2 & & Subject04 & 4 & Subject12 & 3 \\
\hline & Subject05 & 2 & Subject13 & 2 & & Subject05 & 3 & Subject13 & 4 \\
\hline & Subject06 & 2 & Subject14 & 2 & & Subject06 & 3 & Subject14 & 4 \\
\hline & Subject07 & 2 & Subject15 & 2 & & Subject07 & 4 & Subject15 & 3 \\
\hline & Subject08 & 2 & & & & Subject08 & 4 & & \\
\hline
\end{tabular}

The statistical value of the operation force in Condition I was classified as Class 2 and its cluster recognition rate was $93.3 \%$. Only one recognition error that was No.11 subject's confused with Class 4. The statistical value of the operation force in Condition III was classified as Class 1 and its cluster recognition rate was same as Condition I . In this condition, No.15 subject's was confused with Class 2. The statistical value of the operation force in Condition IV was classified as Class 3 and its cluster recognition rate was $86.7 \%$. There were two recognition errors that No.07 and No.15 subjects' were respectively confused with Class 4 and Class 1 . The statistical value of the operation force in Condition II was classified as Class 4 but its cluster recognition rate was only 53.3\% and the lowest. As exampled in Table 2, there were seven recognition errors that the seven subjects' were all confused with Class 3 . The order of the average value of centroids of operation forces was Class $2>$ Class $1>$ Class $4>$ Class 3 . 
Discussion and Suggestion. Analysis of the above statistical results, we found the suspended condition made the recognition rates of Condition II and Condition IV's cluster lower than others by the hybridized K-means. Class 3 and Class 4 were obscured, while Class 1 and Class 2 were classified accurately. So the suspended condition was the most significant effort and testified by Ftest statistic at $1 \%$ significance level. We have known that humans depend on a sophisticated sensory-motor system to sustain proper balance control. Their system is calibrated to terrestrial gravity (G1) and relies on the application of gravitational forces to provide a frame of reference in order to supply a necessary data stream to central neural systems (CNS) located in the brain. [8] Current experimental results support the hypothesis that suspended condition leads to immediate and long term adaptive changes in neural strategies that are used for resolving ambiguous linear accelerations detected by the otolithic system [9].

In the suspended condition experiment, the objects felt as if they were upside-down or might even have difficulty sensing the location of their own arms and legs. About $70 \%$ of objects always adjusted his operation posture and overcame the resistance of the EVA suits in order to operate the handles with maximal force at the beginning of each kind of action. Deductively, the body stability had greater impact on the operation force and operation performance than clothing states. However, while it had been shown that proper suspension had little to no adverse effect on vestibular functions, there was little evidence to suggest that it would permanently correct vestibular and proprioceptive disturbances during space flight. It was also unclear what effects an astronaut might incur when being subjected to only short, multiple periods of this kind of suspension during long duration space flight.

From the perspective of signal shapes, the force signal became stable after about 2.5 seconds that was the time of body stability regulation in suspended condition. When the subjects were in suspended condition, there was a process of adaptation to different operation conditions (in or not in EVA suit) operation direction, operation condition (constrained or suspended). For example, the most difference between Condition I and Condition IV was that in order to exert operation force to the handles, subjects could use the reaction of friction from the ground in Condition I as usual, while they must depend on the reaction of the body from the EVA suits in Condition IV. We found the time point and the number of operations in the process of adaptation was relatively fixed. Perception of location and positions are a result of the brain's ability to integrate visual and auditory signals with vestibular input and proprioceptive information. If these new experimental conditions didn't match fundamental specific calibrated functional patterns in the brain, so called "symptoms of Space Adaptation Syndrome (SAS)" were likely to occur, significantly reducing subject's operational efficiency. And we found the exercises had an important function in postural control that was the coordination of various muscular activities to maintain proper orientation of the body with respect to gravity. Therefore, according to the different types of operation characteristics, multi-operation training and prior operation instructions must improve the subjects' operational efficiency.

\section{Conclusion}

In this work, an ergonomic experiment of four simulated space operation conditions is designed to analyze the mechanical characteristics of basic actions. a hybridized K-means algorithm has been employed, which combines the steps of dimensionality reduction through PCA. Its statistical results show that the suspended operation condition is the most significant effort on the operation force and operation performance. We discuss the experiment phenomenon and their reflection on physiological and cognitive related symptoms being faced by astronauts. Conclusively, we suggest it is most necessary for operation under special circumstances to carry out prior work training and operation instructions.

However, our four condition experiment will not be enough as ground stimulation that strictly stimulated the space operation condition and addressed astronauts' proprioceptive sensory system. Furthermore, data analysis technologies need do some verification and correction works in the future space experiments. 


\section{Acknowledgment}

This work is supported by National Basic Research Program of China (973 Program No.2011CB711000) and Program for Science and Technology on Human Factors Engineering Laboratory.

\section{References}

[1] Amir Ben-Dor, Ron Shamir and Zohar Yakini, “Clustering Gene Expression Patterns,” Journal of Computational Biology, 6(3/4), (1999), p. 281-297.

[2] Chaturvedi J. C. A, Green P, “K-modes clustering,” J. Classification, (18), (2001), p. 35-55.

[3] Daxin Jiang, Chum Tong and Aidong Zhang, "Cluster Analysis for Gene Expression Data," IEEE Transactions on Data and Knowledge Engineering, 16(11), (2004), p. 1370-1386.

[4] Rajashree Dash, Debahuti Mishra, Amiya Kumar Rath, Milu Acharya, "A hybridized K-means clustering approach for high dimensional dataset," International Journal of Engineering, Science and Technology, Vol. 2, No. 2, (2010), p. 59-66.

[5] Dongxu Li, Yan Zhao, "Ergonomics evaluation of three operation postures for astronauts," ICDHM'11 Proceedings of the Third international conference on Digital human modeling, (2011), p. 129-138.

[6] Legner K, "Humans in Space \& Space Biology," Seminars of the United Nations Programme on Space Applications, (2003). http://www.oosa.unvienna.org/.

[7] Young LR, "Examining the Effects of Space Flight on the Human Sensory and Balance System,” Focus on Human Physiology in Space, (2005). Http://www.nsbri.org/HumanPhysSpace/.

[8] P A Souvestre C K Landrock and A P Blaber, "Reducing Incapacitating Symptoms during Space Flight: is Postural Deficiency Syndrome an Applicable Model?” Hippokratia, 12(Suppl 1), (2008) p. 41-48.

[9]Souvestre PA, Blaber AP, Landrock C K. "Space motion sickness: the neurophysiological connection,” 16th IAA Humans in Space Symposium proceedings; Beijing, China. (2007), p. 145146. 